\title{
Single-walled carbon nanohorn aggregates promotes mitochondrial dysfunction-induced apoptosis in hepatoblastoma cells by targeting SIRT3
}

\author{
BOWEI LI ${ }^{1,2^{*}}$, XIAOXUN CHEN ${ }^{3 *}$, WENBIN YANG ${ }^{4}$, JINGLIANG HE $^{5}, \mathrm{KE} \mathrm{HE}^{2}$, \\ ZHENGLIN XIA $^{2}$, JINQIAN ZHANG ${ }^{6 *}$ and GUOAN XIANG ${ }^{1,2^{*}}$
}

\begin{abstract}
${ }^{1}$ Department of Postgraduate Studies, The Second Clinical College of Southern Medical University, Guangzhou, Guangdong 510515; ${ }^{2}$ Department of General Surgery, Guangdong Second Provincial General Hospital, Guangzhou, Guangdong 510317; ${ }^{3}$ Department of Gastrointestinal Surgery, The Guigang City People's Hospital, Guigang, Guangxi 537100; ${ }^{4}$ Department of General Surgery, The Second Affiliated Hospital of Xi'an Jiao Tong University, Xi'an, Shaanxi 710004; ${ }^{5}$ Department of General Surgery, Shunde Hospital of Guangzhou University of Chinese Medicine, Foshan, Guangdong 528300; ${ }^{6}$ Department of Laboratory Medicine, Guangdong Second Provincial General Hospital, Guangzhou, Guangdong 510317, P.R. China
\end{abstract}

Received March 8, 2018; Accepted June 11, 2018

DOI: 10.3892/ijo.2018.4459

\begin{abstract}
Single-walled carbon nanohorns (SWNHs) can accumulate in a variety of cell types or tissues and exert biological effects, which have been demonstrated to induce apoptosis in hepatoblastoma cells. However, the role and molecular mechanisms of SWNHs remain unclear. The mitochondrion is an important subcellular structure and may contribute to apoptosis that is induced by SWNHs in hepatoblastoma cells. To address this question, the mitochondrial function of HepG2 or L02 cells that were treated with SWNHs was examined. The results indicated that SWNHs were able to decrease the mitochondrial membrane potential and suppress the activity of the $\mathrm{Na}^{+} / \mathrm{K}^{+}$-ATPase. Secondly, HepG2 cells and L02 cells were treated with SWNHs in vivo and in vitro. The expression of mitochondrial-associated proteins [acyl-CoA synthetase short chain family member 1 , Bax, cytochrome C (CYT-C), sodium channel epithelial $1 \alpha$ subunit, sirtuin 3 (SIRT3) and voltage-dependent anion channel 1] was analyzed by western blotting and immunohistochemical staining. The results
\end{abstract}

Correspondence to: Professor Guoan Xiang, Department of General Surgery, Guangdong Second Provincial General Hospital, 466 Xingang Middle Road, Guangzhou, Guangdong 510317, P.R. China

E-mail: guoan_66@163.com

Dr Jinqian Zhang, Department of Laboratory Medicine, Guangdong Second Provincial General Hospital, 466 Xingang Middle Road, Guangzhou, Guangdong 510317, P.R. China

E-mail: jingwanghou@163.com

${ }^{*}$ Contributed equally

Key words: single-walled carbon nanohorns, hepatoblastoma, apoptosis, mitochondria, sirtuin 3 revealed that SWNH treatment was able to alter the expression of multiple mitochondrial apoptotic pathway-associated proteins in HepG2 cells. SWNH treatment was able upregulate the expression of SIRT3, CYT-C and VDAC1 and downregulate the expression of AceCS2, but it had a more stable effect on SIRT3. However, similar findings were not observed in L02 cells. Therefore, the data from the present study indicated that SWNHs might be used as a safe anticancer agent, where it is able to trigger mitochondrial dysfunction-induced apoptosis by upregulating SIRT3 expression in HepG2 cells.

\section{Introduction}

Hepatoblastoma, originating in hepatic primordial embryonic cells, is the most common malignancy of primary epithelial origin in children worldwide and is usually diagnosed in the first 3 years of life (1-3). For patients with hepatoblastoma, the majority of cases are sporadic and no predisposition is identified, so the survival rate usually depends on the resectability of the tumor (4). Fortunately, chemotherapy is able to shrink unresectable tumors to a resectable size, but the toxicity of chemotherapy drugs to normal tissues limits their use (5). Furthermore, the poor results of multifocal disseminated and metastatic tumors merit investigation of new cytotoxic drugs and substances against specific molecular targets.

Lately, a number of researchers have reported that sirtuin 3 (SIRT3) has a major role in repairing cell injury, inhibiting cell necrosis and preventing cell canceration (6-8). As one of the seven families of the sirtuin gene, SIRT3 is primarily localized in the mitochondria and it exhibits histone deacetylase activity. SIRT3 has a crucial role in cell development and repair (9). For example, SIRT3 is necessary to prevent age-associated hearing loss, reduce oxidative stress and mediate damage by caloric restriction $(10,11)$. The mechanism that SIRT3 attenuates the dysfunction of activated cells is closely associated with 
mitochondrial function, which includes decreasing the opening of the mitochondrial permeability transition pore (MPTP) and expression of cyclophilin D as well as inhibiting the release of CYT-C to the cytoplasm, and decreasing the Bax/B-cell lymphoma-2 (Bcl-2) ratio and caspase-3/9 activity (7). On the contrary, the deficiency of SIRT3 leads to the failure of repair from damage in cells, and continuous injury is able to induce carcinogenesis $(8,12)$. SIRT3 is able to function as a cytoprotective factor and mitochondrial tumor suppressor (12), and therefore upregulating SIRT3 expression may be a potential anticancer strategy for tumor therapy.

The single-walled carbon nanohorn (SWNHs), a graphenebased pseudo tubule with the absence of any metal catalyst, was first synthesized by Iijima's group in 1999 (13). Based on the characteristics of good biocompatible and potential application for drug carriers, SWNHs had quickly entered the biomedicine field $(14,15)$. Unexpectedly, SWNHs are able to exert biological effects, including anticancer (16) and antiinflammatory activity (17). In addition, SWNHs were able to restrain growth, proliferation and mitotic entry of human liver normal and cancer cells. SWNHs were also able to inhibit cell apoptosis, particularly in the HepG2 cell line, which was revealed by previous findings (18). SWNHs have been demonstrated to promote apoptosis in HepG2 cells by activating tumor suppressor p53, caspase-3 and -7 (18). However, there is a lack of understanding regarding the upstream molecular mechanism of SWNHs and its effect on subcellular structures. Therefore, to the best of our knowledge, the present study is the first description of the association between treatment with SWNHs and mitochondrial function.

\section{Materials and methods}

Properties of SWNHs. SWNHs were produced in air by arcdischarge, and then high-resolution transmission electron microscopy was used for characterization. Subsequently, the SWNHs were suspended in ultrapure water $(10 \mu \mathrm{g} / \mathrm{ml})$, placed on a polystyrene dish and dried at $80^{\circ} \mathrm{C}$ for $4 \mathrm{~h}$. The dishes were treated with ultraviolet irradiation for 1-2 $\mathrm{h}$ before use. The abbreviations (SW0, SW10, SW20, SW30, SW40 and SW50) correspond to the concentrations of SWNHs in each dish: $0,0.21,0.42,0.64,0.85$ and $0.96 \mu \mathrm{g} / \mathrm{cm}^{2}$, respectively.

Cell culture. The human hepatoblastoma cell line, HepG2, and the human normal liver cell line, L02, were obtained from American Type Culture Collection (Manassas, VA, USA). The cells were seeded into PS dishes coated with SW0, SW10, SW20, SW30, SW40 and SW50 and then cultured with Dulbecco's modified Eagle's medium accompanied with $10 \%$ fetal bovine serum (Gibco; Thermo Fisher Scientific, Inc., Waltham, MA, USA), $100 \mathrm{U} / \mathrm{ml}$ penicillin $\mathrm{G}$ and $100 \mu \mathrm{g} / \mathrm{ml}$ streptomycin (SW30010; BD Biosciences, San Jose, CA, USA) at $5 \% \mathrm{CO}_{2}$ with $37^{\circ} \mathrm{C}$.

Mitochondrial membrane potential assay. Mitochondrial membrane potential (MMP) was analyzed using a JC-1 fluorescent probe kit (Beyotime Institute of Biotechnology, Haimen, China). The lipophilic and cationic fluorescent dye, JC-1 (5,5',6,6'-tetrachloro-1,1',3,3'-tetraethyl benzimidazole carbocyanine iodide), is capable of selectively entering mitochondria, forming aggregates in mitochondria and emitting red fluorescence at high MMP. However, in low MMP, JC-1 cannot enter mitochondria and forms monomers that emit green fluorescence. The ratio of red to green fluorescence provides an estimate of change in MMP. The aggregates/monomer ratio was quantified at an excitation wavelength of $524 \mathrm{~nm}$, emission wavelength of $590 \mathrm{~nm}$ (monomer, excitation at $490 \mathrm{~nm}$ and emission at $530 \mathrm{~nm}$ ). HepG2 cells or L02 cells were co-cultured with SWNHs for $48 \mathrm{~h}$ in a 6-well plate and then treated with $\mathrm{JC}-1(5 \mu \mathrm{g} / \mathrm{ml})$ at $37^{\circ} \mathrm{C}$ for $30 \mathrm{~min}$. Then, the cells were washed with JC-1 Staining Buffer (Beyotime Institute of Biotechnology) for three times. The fluorescence intensity was immediately measured using a fluorescence microscope at a magnification of x200 (IX71; Olympus Corporation, Tokyo, Japan). The intensity of green and red fluorescence was measured using Image-Proplus 6.0 software (Media Cybernetics, Rockville, MD, USA).

$\mathrm{Na}^{+} / \mathrm{K}^{+}$-ATPase activity study. $\mathrm{Na}^{+} / \mathrm{K}^{+}$-ATPase activity was identified as the difference between inorganic phosphates $(\mathrm{Pi})$ released and determined using a $\mathrm{Na}^{+} / \mathrm{K}^{+}$-ATPase assay kit (Nanjing Jiancheng Bioengineering Institute, Nanjing, China). Briefly, following treatment with SWNHs in $37^{\circ} \mathrm{C}$ for $48 \mathrm{~h}$, HepG2 cells were harvested and physiological saline was added. After three rounds of repeated freezing $\left(-80^{\circ} \mathrm{C}\right)$ and thawing, the suspensions of homogenates were centrifuged at $8,000 \mathrm{x} \mathrm{g}$ and $4^{\circ} \mathrm{C}$ for $15 \mathrm{~min}$ to obtain the supernatant and then the protein concentration was evaluated using BCA protein assay (Thermo Fisher Scientific, Inc.). Finally, the $\mathrm{Na}^{+} / \mathrm{K}^{+}$-ATPase activity was determined by measuring the amount of inorganic phosphate with malachite green dye, and the results were expressed as micromoles per milligrams of protein.

Western blotting of HepG2 and LO2 cells. HepG2 $\left(3 \times 10^{5}\right)$ and L02 $\left(3 \times 10^{5}\right)$ cells were seeded into dishes and co-cultured with or without SWNHs for $48 \mathrm{~h}$. Then, total protein was extracted with a protein extraction kit (Nanjing KeyGen Biotech Co., Ltd., Nanjing, China). The concentration of these proteins was monitored by the BCA protein assay (Thermo Fisher Scientific, Inc.). The proteins extracted from each sample were subjected to $10 \%$ SDS-PAGE electrophoresis and then transferred to a PVDF membrane (Merck Millipore, Darmstadt, Germany). Next, the membranes were incubated with primary antibodies [including acetyl-CoA synthetase 2 (AceCS2) (DF3727, 1:200), SCNN1A (DF9199, 1:200), SIRT3 (AF5135, 1:200), VDAC1 (DF6140, 1:200) (all from Affinity Biosciences, Cincinnati, OH, USA), Cyt-C (sc-13561, 1:50), Bax (sc-4239, 1:50) (both from Santa Cruz Biotechnology, Inc., Santa Cruz, CA, USA)] and secondary antibodies (S0001, 1:5,000; Affinity Biosciences) at $4^{\circ} \mathrm{C}$ overnight. The blots were projected onto X-ray film (Carestream, Shanghai, China) following visualization by chemiluminescence using the ECL kit (EMD Millipore, Billerica, MA, USA). Image Pro Plus 6.0 software (Media Cybernetics) was used to measure the intensity of protein bands.

In vivo experiment. A total of 20 nude mice, half of male and female, were purchased from the Laboratory of Animal Sciences (Southern Medical University, Guangzhou, China). 
The nude mice were randomly divided into 2 groups, and the number of males and females in each group was guaranteed to be equal. The experiment was initiated with 6 week-old mice weighing 20-25 g. HepG2 cells were resuspended at a density of $1 \times 10^{7}$ cells $/ \mathrm{ml}$, and then $0.1 \mathrm{ml}$ suspension was subcutaneously injected into the right flanks of nude mice ( $n=10 /$ group). While the tumor size reached $3-8 \mathrm{~mm}$, it was set as the first day of the experiment.

SWNHs were formulated at a concentration of $1.0 \mathrm{mg} / \mathrm{ml}$ in PBS. The nude mice were randomly divided into two groups and then injected with SWNHs $(50 \mu \mathrm{l})$ or vehicle once every three days for 6 times, and this was terminated on the 18th day. Then, the mice were sacrificed on the 21st day, and the tumor was dissected from each mouse. These tumors were cut into two sections, then the expression of SIRT3, AceCS2, SCNN1a, VDAC1, CYT-C and Bax was analyzed by western blotting and immunohistochemical staining assay. Staining with $\mathrm{H} \& \mathrm{E}$ and Sirius red was performed to observe cell morphology and distribution of extracellular collagen fibers. After the sections were deparaffinized and hydrated, tumor tissues were H\&E-stained using a hematoxylin and eosin staining kit (Solarbio, Beijing, China); the nuclei were stained with hematoxylin stain at $37^{\circ} \mathrm{C}$ for $5 \mathrm{~min}$, and the cytoplasm was then stained with eosin staining solution at $37^{\circ} \mathrm{C}$ for $30 \mathrm{sec}$. The extracellular matrix was stained with Sirius Red stain (Solarbio, Beijing, China) at $37^{\circ} \mathrm{C}$ for $15 \mathrm{~min}$.

The experiments on the mice were performed according to the standards that were supported by the Animal Protection Committee of Southern Medical University. The ethics approval was obtained from Animal Ethical and Welfare Committee of South Medical University (reference no. IACUC-2017-0120).

Statistics analysis. Data were obtained from at least three independent experiments. The data are presented as the mean \pm standard deviation. One-way analysis of variance or Student's t-test was used for statistical analysis with SPSS (version 20.0; IBM Corp., Armonk, NY, USA). For multiple comparisons of variance analysis, different post hoc test methods were chosen based on the homogeneity of variance. When the variances were equal, the LSD test was used. When the variance was not uniform, Dunnett T3 was used. $\mathrm{P}<0.05$ was used to indicate statistical significance. GraphPad Prism software (GraphPad Software, Inc., La Jolla, CA, USA) was used.

\section{Results}

Treatment with SWNHs depolarizes MMP of HepG2 cells. Mitochondria have a central role in the regulation of apoptosis as it contains many apoptogenic proteins, including CYT-C and apoptosis-inducing factor (AIF). Depolarized MMP may change mitochondrial permeability transition (MPT), which results in the release of apoptogenic proteins $(19,20)$. To demonstrate the effect of SWNHs on MMP, the JC-1 kit was used to detect the changes in MMP in L02 and HepG2 cells. The results indicated that after the co-culturing of the cells with SWNHs for $48 \mathrm{~h}$, there was no observable change in MMP in L02 cells (Fig. 1). As for the HepG2 cells, MMP was depolarized in a concentration-dependent manner. The difference between the experimental group and the control group was statistically significant, when the concentration of SWNHs was $>0.64 \mu \mathrm{g} / \mathrm{cm}^{2}(\mathrm{P}<0.05)$. This result showed that SWNHs, as a type of nanomaterial, were able to enter and target mitochondria to induce mitochondrial dysfunction in tumor cells. However, it has little effects on normal cell mitochondrial function.

SWNHs inhibited $\mathrm{Na}^{+} / \mathrm{K}^{+}$-ATPase activity of HepG2 cells. The $\mathrm{Na}^{+} / \mathrm{K}^{+}$-ATPase, a membrane ATP consumable protein, is critically important for the maintenance of the ion gradients required for cell homeostasis (21). The $\mathrm{Na}^{+} / \mathrm{K}^{+}$-ATPase activity of HepG2 cells was detected after co-culture with SWNHs for $48 \mathrm{~h}$. The result showed low doses of SWNHs $\left(<0.64 \mu \mathrm{g} / \mathrm{cm}^{2}\right)$ have little effects on the activity of $\mathrm{Na}^{+} / \mathrm{K}^{+}$-ATPase (Fig. 2). However, with an increasing concentration of SWNHs, the activity of $\mathrm{Na}^{+} / \mathrm{K}^{+}$-ATPase gradually declined. When the concentration was $\geq 0.85 \mu \mathrm{g} / \mathrm{cm}^{2}$, the activities of the experimental groups were statistically significant compared with the control group $(\mathrm{P}<0.05)$.

SWNHs induce mitochondrial apoptosis pathway-related protein expression in vitro. To confirm whether mitochondrial dysfunction-induced apoptosis in HepG2 and L02 cells appeared after exposure to SWNHs, the expression of SIRT3 and other five mitochondrial apoptosis markers was determined by western blotting. In HepG2 cells, SWNHs were able to upregulate the expression of SIRT3, VDAC1, SCNN1A and CYT-C (Fig. 3A and C). In particular, the expression levels of CYT-C, SIRT3 and VDAC1 exhibited 2 or 3-fold increase in expression when compared with the control group, and these differences in expression were statistically significant $(\mathrm{P}<0.05)$. Furthermore, a high concentration of SWNHs was able to downregulate the expression of Bax and AceCS2. When the concentration of SWNHs was $\geq 1 \mu \mathrm{g} / \mathrm{cm}^{2}$, the expression of Bax was significantly different from that of the control group $(\mathrm{P}<0.05)$. When the concentration of SWNHs was $\geq 0.85 \mu \mathrm{g} / \mathrm{cm}^{2}$, there was a difference in the expression of AceCS2 compared with the control group $(\mathrm{P}<0.05)$. In L02 cells, the expression of CYT-C, SIRT3, VDAC1, AceCS2, Bax and SCNN1A group did not exhibit significant changes compared with the control (Fig. 3B and D). This result was a preliminary validation of our hypothesis that SWNHs are able to induce the apoptosis of HepG2 cells via the mitochondrial apoptotic pathway.

SWNHs induce the expression of mitochondrial apoptosis pathway-associated proteins in vivo. To further confirm whether SWNHs are able to induce apoptosis that is mediated by mitochondrial dysfunction in vivo, nude mice bearing HepG 2 tumor xenografts were employed. After 21 days of treatment with SWNHs, tumors were dissected from each mouse (Fig. 5B). The expression of mitochondrial apoptosis pathway-associated proteins in the xenografts was analyzed using western blotting and immunohistochemical staining. Western blotting revealed that when compared with the control group, SWNH treatment was able to upregulate the expression of CYT-C, Bax, SCNN1A, AceCS2, SIRT3 and VDAC1 (Fig. 4). However, only differences in the expression of SIRT3 and Bax between the treatment and control groups were statistically significant $(\mathrm{P}<0.05)$. For immunohistochemical 
HepG2

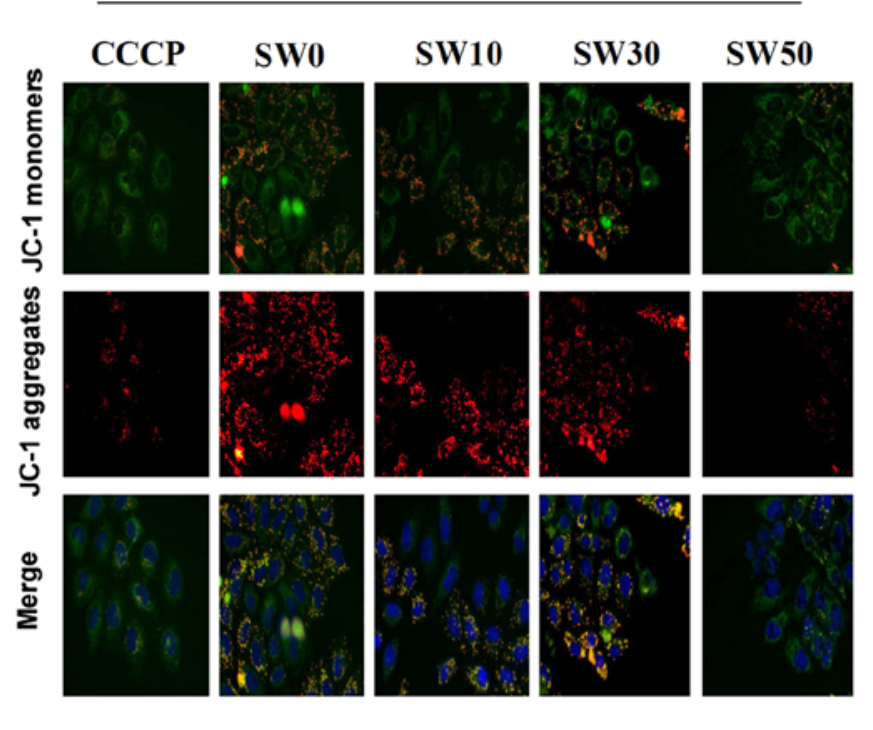

L02

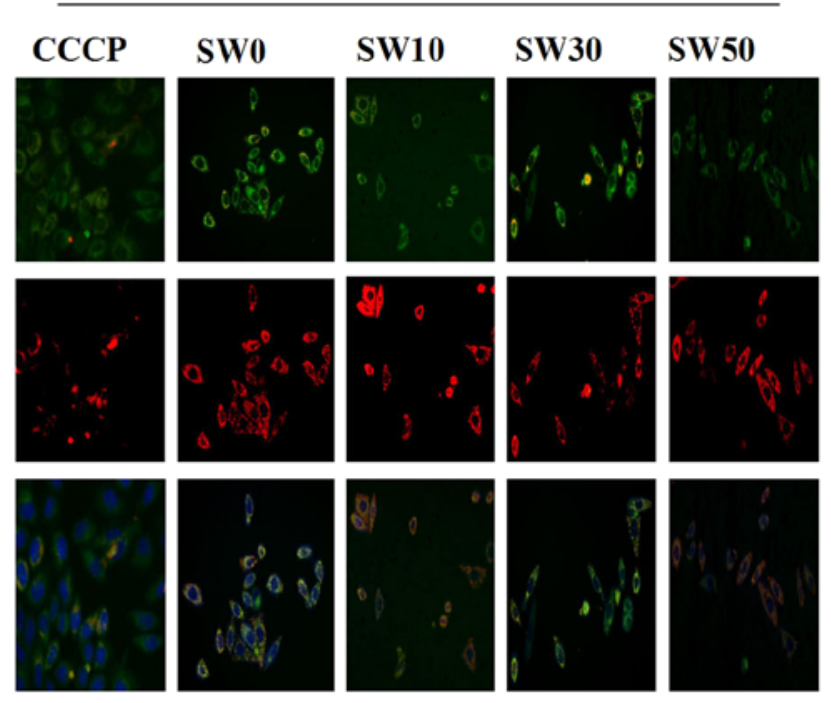

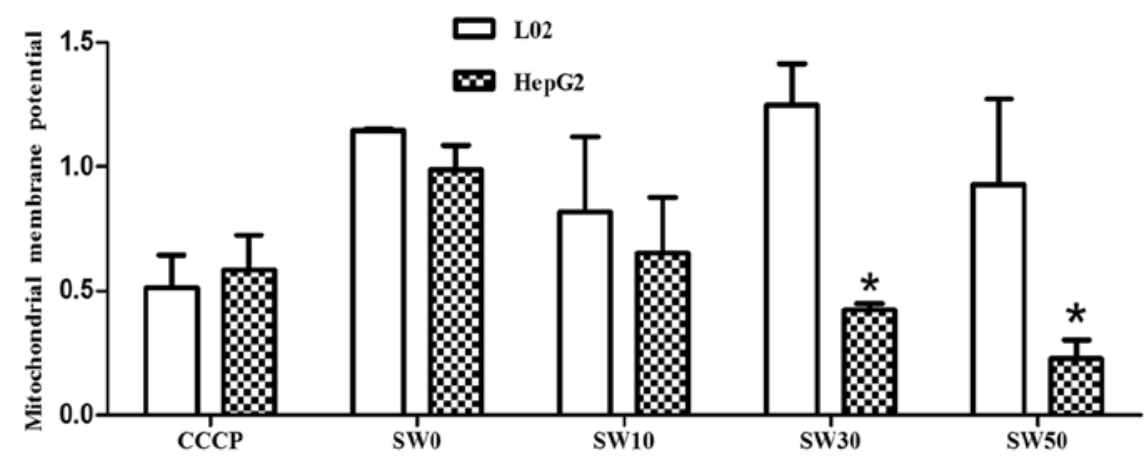

Figure 1. SWNHs depolarizes MMP in HepG2 cells. Following treatment with SWNHs in 6-well plates for $48 \mathrm{~h}$, the JC-1 detection kit was used to determine the MMP of HepG2 and L02 cells. The nuclei were stained with Hoechst 33342. CCCP is a positive control group. SW0, SW10, SW20, SW30, SW40 and SW50 correspond to the concentrations of SWNHs in each dish: $0,0.21,0.42,0.64,0.85$ and $0.96 \mu \mathrm{g} / \mathrm{cm}^{2}$, respectively. The images were captured using a fluorescence microscope. Magnification, x200. The intensity of green and red fluorescence was measured using Image-Proplus 6.0 software. The data are presented as the mean \pm standard deviation $(\mathrm{n}=3)$. ${ }^{*} \mathrm{P}<0.05$ vs. CCCP. MMP, mitochondrial membrane potential; SWNH, single-walled carbon nanohorn.

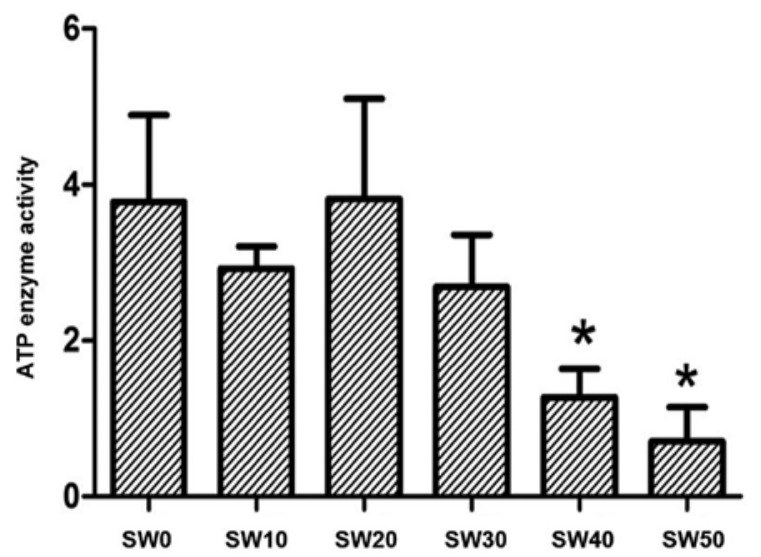

Figure 2. SWNHs inhibit the $\mathrm{Na}^{+} / \mathrm{K}^{+}$-ATPase activity of HepG2. Following co-culture with SWNHs for $48 \mathrm{~h}$, the $\mathrm{Na}^{+} / \mathrm{K}^{+}$-ATPase of HepG2 was analyzed. Data are presented as the mean \pm standard deviation $(n=3)$. ${ }^{*} \mathrm{P}<0.05$ vs. the SW0 group. SW0, SW10, SW20, SW30, SW40 and SW50 correspond to the concentrations of SWNHs: $0,0.21,0.42,0.64,0.85$ and $0.96 \mu \mathrm{g} / \mathrm{cm}^{2}$, respectively. SWNH, single-walled carbon nanohorn.

staining results, SIRT3 expression in the treatment group was significantly higher compared with the control group.
There were differences between immunohistochemical staining and western blotting in some places (Fig. 5A and D). Immunohistochemical staining indicated that there was a decreased expression of AceCS2 following treatment with SWNHs, which was statistically significant $(\mathrm{P}<0.05)$. By contrast, western blotting indicated an decline in AceCS2 expression. Furthermore, immunohistochemical staining revealed that SWNHs were able to upregulate VDAC1 expression $(\mathrm{P}<0.05)$, but western blotting indicated a decrease in VDAC1 expression.

\section{Discussion}

Apoptosis is an ordered and coordinated process of cellular change that occurs under physiological or pathological conditions. Apoptosis has an important role in the occurrence and development of tumor. Furthermore, inhibition of apoptosis is the principal mechanism of tumorigenesis (22). To date, a number of apoptotic pathways have been identified. One of the most important of apoptotic pathways is the intrinsic mitochondria-dependent pathway (23). The mitochondrion is a double-membrane organelle that participates in various functions, including ATP production, steroid hormone synthesis, 
A

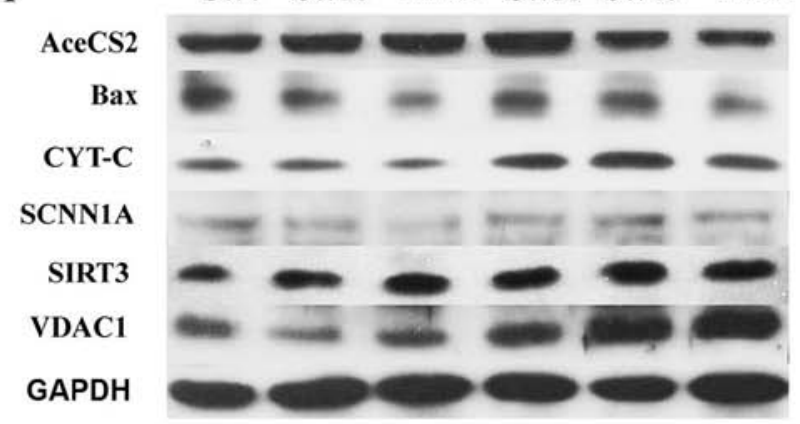

B

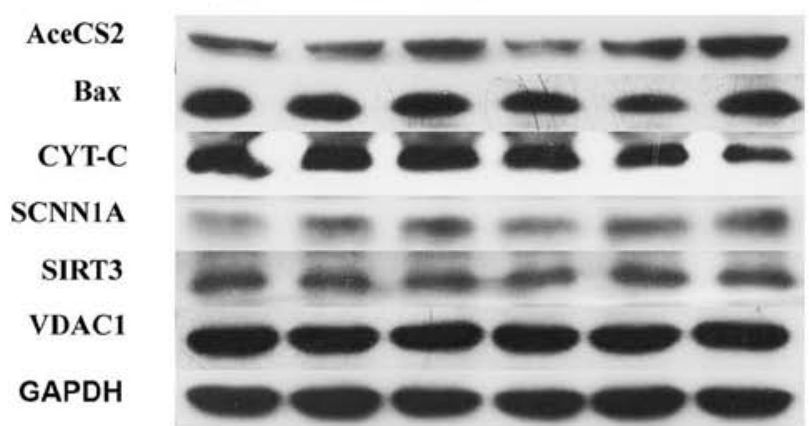

C

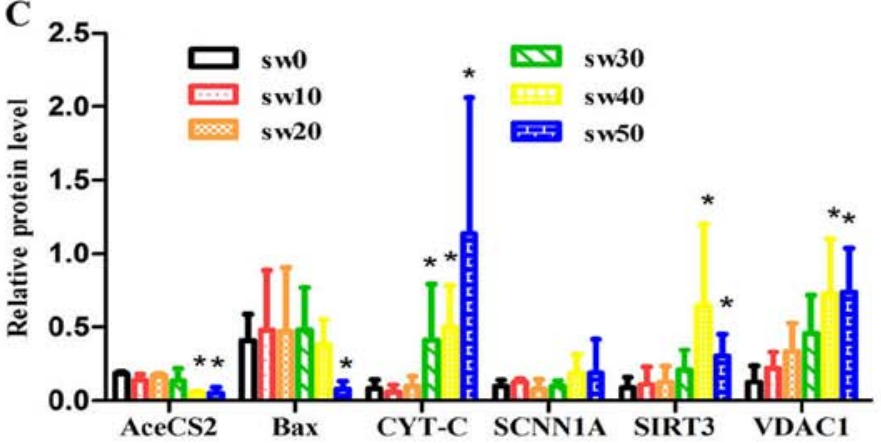

D

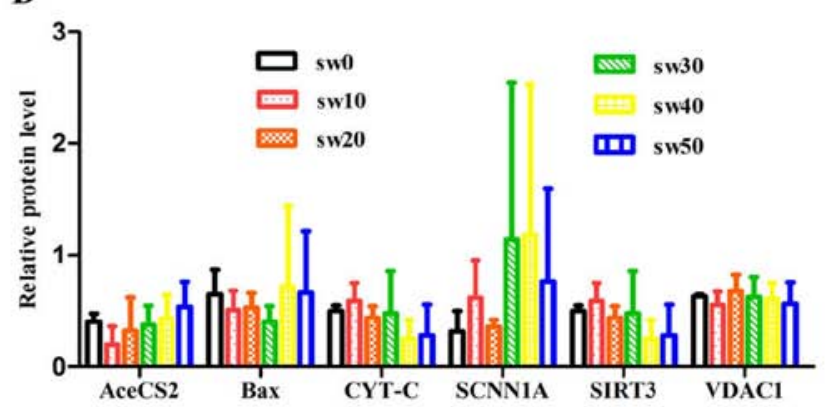

Figure 3. Treatment with SWNHs alters the expression of mitochondrial apoptotic pathway-associated proteins in vitro. (A) HepG2 and (B) L02 cells were incubated with different concentrations of SWNHs in 6-well plates for $48 \mathrm{~h}$. The changes in the expression and the relative quantification of proteins in (C) HepG2 cells or (D) L02 cells were identified by western blotting. SW0, SW10, SW20, SW30, SW40 and SW50 correspond to the concentrations of SWNHs: $0,0.21,0.42,0.64,0.85$ and $0.96 \mu \mathrm{g} / \mathrm{cm}^{2}$, respectively. Data are presented as the mean \pm standard deviation (n=3). $\mathrm{P}<0.05$ compared with the SW0 group. AceCS2, acyl-CoA synthetase short chain family member 1; SCNN1A, sodium channel epithelial 1 $\alpha$ subunit; SIRT3, sirtuin 3; SWNH, single-walled carbon nanohorn; VDAC1, voltage-dependent anion channel 1.

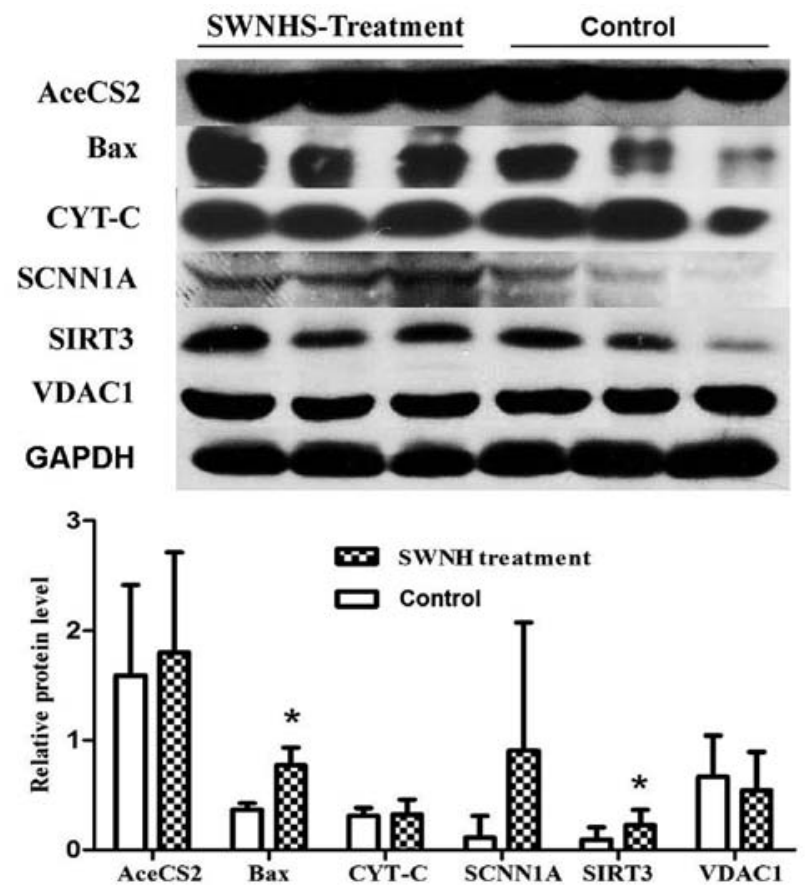

Figure 4. Treatment with SWNHs alters the expression of mitochondrial apoptosis pathway-associated proteins in vivo. HepG2 xenografts in nude mice were regularly treated with SWNHs. The changes in expression and relative quantification of mitochondrial apoptosis pathway-associated proteins that were induced by SWNHs as detected by western blotting in HepG2 xenografts. Data are presented as the mean \pm standard deviation $(\mathrm{n}=3) . \mathrm{P}<0.05$ compared with the control group. AceCS2, acyl-CoA synthetase short chain family member 1; SCNN1A, sodium channel epithelial $1 \alpha$ subunit; SIRT3, sirtuin 3; SWNH, single-walled carbon nanohorn; VDAC1, voltage-dependent anion channel 1. reactive oxygen species (ROS) production and ionic regulation (24). The intrinsic mitochondria-dependent pathway converges at the membrane of the mitochondria. Under certain cell death signals, the outer membrane of the mitochondria becomes sufficiently permeable to allow the release of soluble hemoprotein CYT-C, AIF, Smac/Diablo, endonuclease G, Bax as well as other intermembrane space proteins, which irreversibly activate downstream caspases to carry out the apoptosis process (25).

Difficulty in treating hepatoblastoma is a worldwide problem, which is characterized by a high prevalence of drug resistance and is able to be cured by surgical resection $(26,27)$. Systemic chemotherapeutic, as a preoperative routine treatment for unresectable hepatoblastoma, has been applied in clinical practice for many years (28). However, a high toxicity and drug resistance pose challenges to the efficiency of chemotherapeutic drugs (29). Therefore, it is extremely important to find more effective chemotherapeutics.

Nanomaterials are likely to have an important role in the treatment of tumors, particularly for hepatoblastoma. Preliminary experimental studies have shown that a number of carbon nanomaterials inhibited the growth and proliferation of L02 or HepG2 cells $(30,31)$. SWCNTs have great potential for tumor treatment due to their excellent biocompatibility and huge drug-bearing capacity. SWCNTs have been demonstrated to enter cells in different ways and are located in different subcellular structures, including lysosomes, mitochondria and even the nuclei (32). SWNHs have similar structure to SWCNTs, but SWNHs have several unique properties, including being non-toxic and having a high capacity 

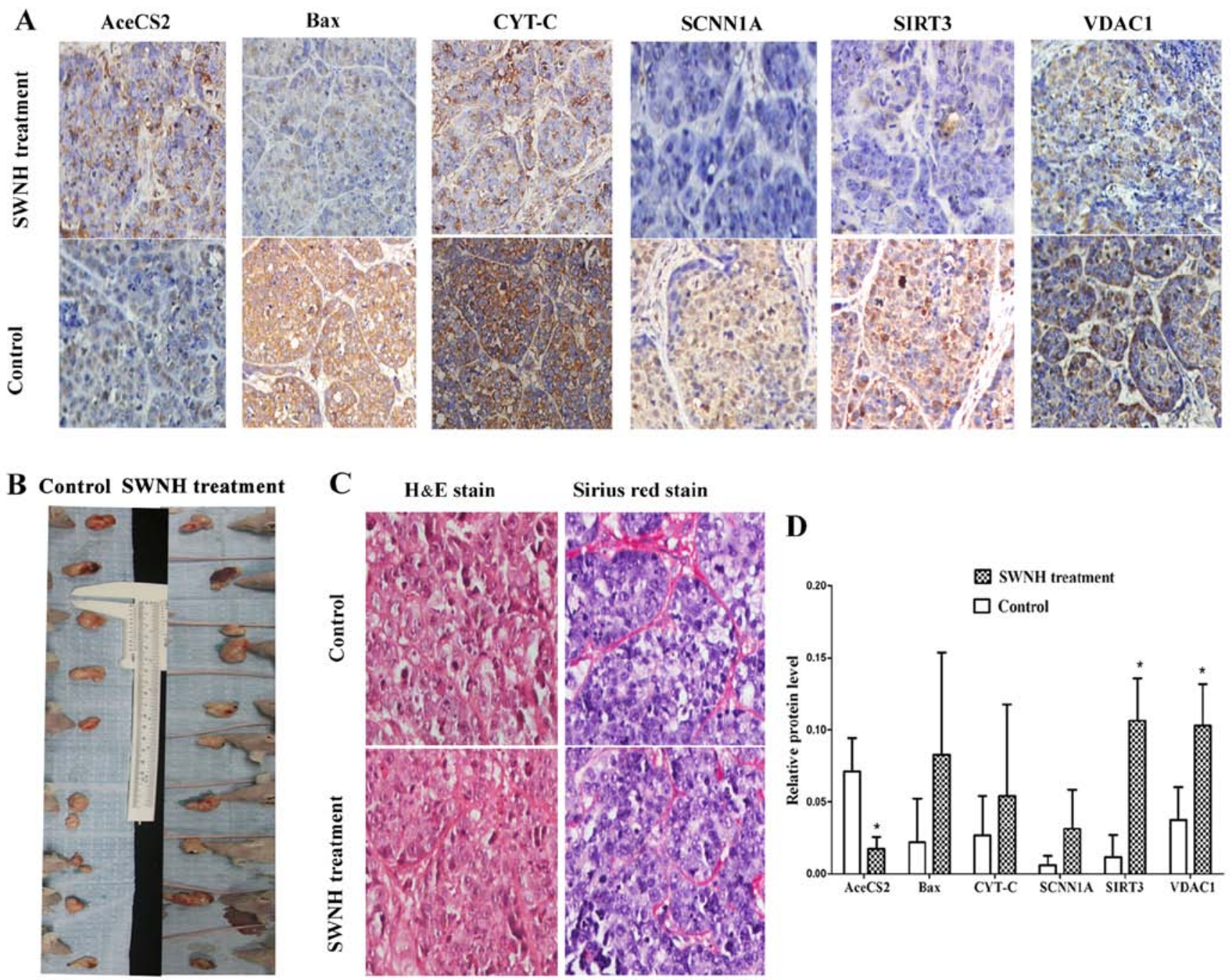

D

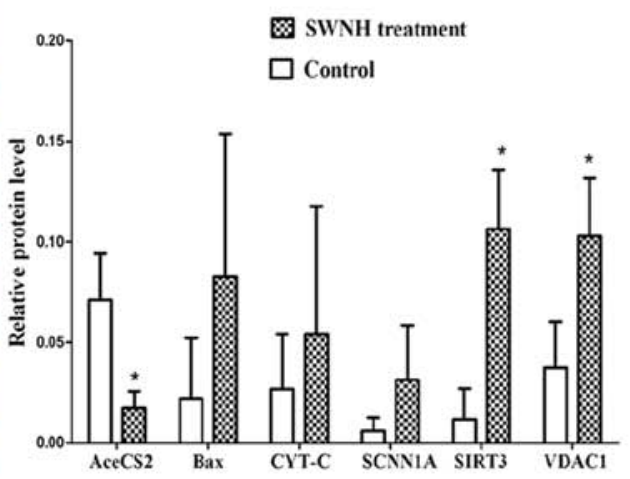

Figure 5. Treatment with SWNHs alters the expression of mitochondrial apoptosis pathway-associated proteins in HepG2 xenografts in vivo. (A) The changes in the expression of mitochondrial apoptosis pathway-associated proteins was detected by immunohistochemistry in the SWNH treatment group and control group. The cells were observed and photographed at a magnification of $\mathrm{x} 200$ via a contrast microscope. (B) A tumor was dissected from each mouse. (C) The morphology of the nuclei, cytoplasm and collagen fibers in the HepG2 xenograft were observed by H\&E staining and Sirius red staining at a magnification of x200 via a contrast microscope. (D) Relative quantification of mitochondrial apoptosis pathway-associated proteins in the SWNH treatment group and control group. " $\mathrm{P}<0.05$ vs. the control group. Data are presented as the mean \pm standard deviation $(\mathrm{n}=3)$. AceCS2, acyl-CoA synthetase short chain family member 1 ; SCNN1A, sodium channel epithelial 1 $\alpha$ subunit; SIRT3, sirtuin 3; SWNH, single-walled carbon nanohorn; VDAC1, voltage-dependent anion channel 1.

for drug loading (13). SWNHs have been considered as an excellent candidate for drug carrier (33). Notably, SWNHs itself has a certain biological effect. It has been demonstrated that SWNHs are able to induce the apoptosis of HepG2 cells, while they have little effects on the normal hepatic cell line L02 (18). Although the mechanism of how SWNHs promote the apoptosis of HepG2 cells and the subcellular localization of SWNHs remain unclear, the mechanism may be similar to SWCNTs, which can affect the function of mitochondria (32).

The maintenance of MMP has an important role in mitochondrial function, and the present results of MMP detection suggested that SWNHs might be able to depolarize the MMP of HepG2 cells. There might be two reasons for this observation. Firstly, SWNHs accumulate in the mitochondrial matrix, and change the distribution of protons and ions on both sides of the mitochondrial inner membrane, which might be a direct reason for MMP dipolarization. Secondly, since SIRT3 has been demonstrated to have an important role in the integrity of the mitochondrial membrane, changes in SIRT3 expression may also have an impact on MMP (34). However, there were no marked changes in the MMP of L02 cells in this study. The differences in the biological behavior of HepG2 and L02 may be associated with the endocytosis and metabolic rate of cells. The loss of MMP is able to reduce the permeability of the mitochondrial membrane, which not only means the loss of mitochondrial function but also the early manifestation of apoptosis (35).

The mitochondrion is the center of cellular energy production. The depolarization of the mitochondrial membrane may block the oxidative phosphorylation of cells, thereby affects ATP synthesis. Nevertheless, SWNHs not only affects the synthesis of ATP but also restricts the ability of the cells to utilize ATP. The result of $\mathrm{Na}^{+} / \mathrm{K}^{+}$-ATPase activity detection presented that low concentrations of SWNHs had no significant effects on the activity of the ATP enzyme. However, with the increase in the concentration of SWNHs, the activity 
Pathway of SWNHs Affecting Mitochondrial Function and Apoptosis in HepG2 Cells

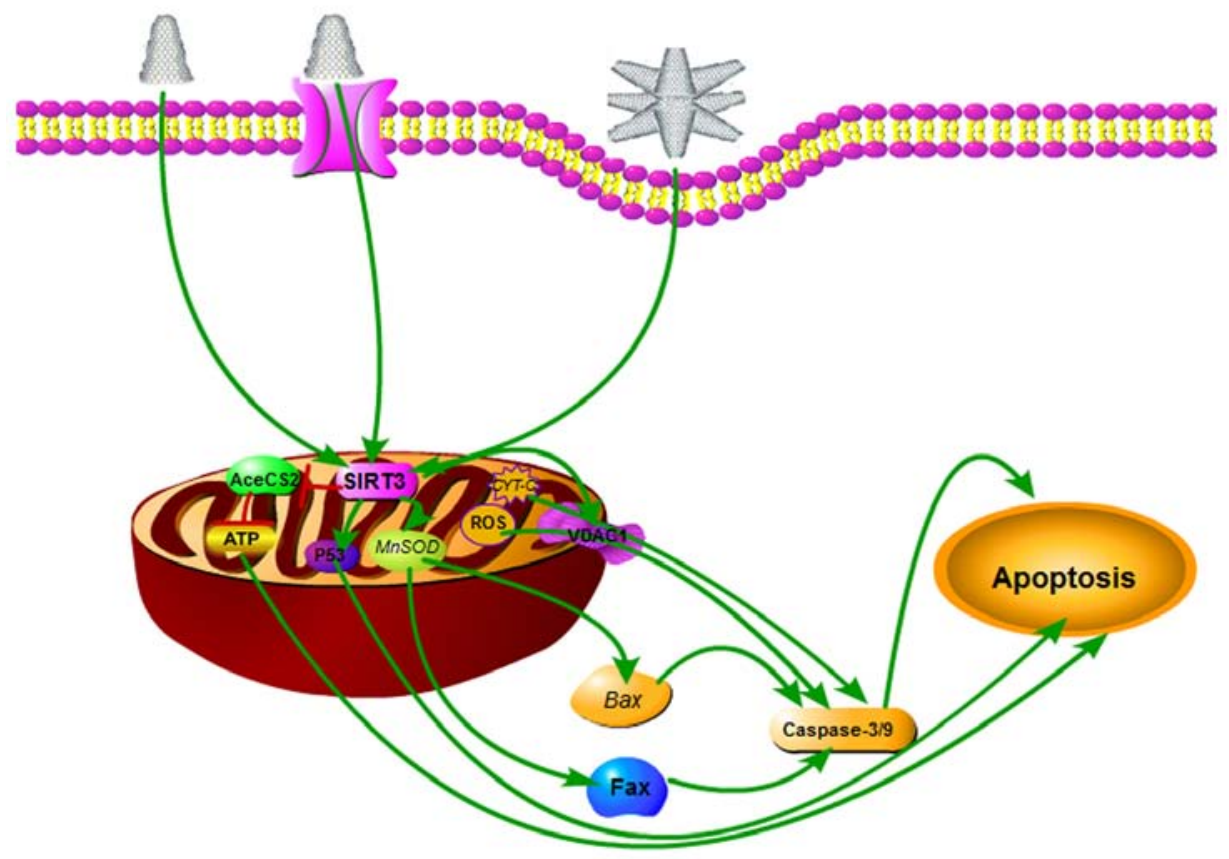

Figure 6. SWNHs affect mitochondrial function and apoptosis in HepG2 cells. SWNH monomers can enter cells by diffusion or ion channels, while SWNH polymers enter cells via endocytosis. Intracellular SWNHs can accumulate in the mitochondria and upregulate the expression of SIRT3 and VDAC1. The activation of SIRT3 subsequently causes the release of CYT-C, Bax and p53 into the cytoplasm, activate the caspase cascade and induce apoptosis. At the same time, SIRT3 is able to inhibit the expression of AceCS2, reduce intracellular ATP production and promote cell apoptosis. AceCS2, acyl-CoA synthetase short chain family member 1; CYT-C, cytochrome C; SIRT3, sirtuin 3; SWNH, single-walled carbon nanohorn; VDAC1, voltage-dependent anion channel 1.

of the ATP enzyme decreased gradually. This implied that higher concentrations $\left(\geq 0.85 \mu \mathrm{g} / \mathrm{cm}^{2}\right)$ of SWNHs were able to inhibit the ability of the HepG2 cells to use ATP, and it led to a decrease in cell metabolism. It was also revealed that SWNHs were able to enter the cytoplasm via the cell membrane and disrupt cellular energy metabolism, which is another hallmark of apoptosis.

To confirm the mechanism of the apoptosis pathway that is induced by mitochondrial dysfunction in SWNH-treated cells, the expression of mitochondrial apoptosis pathway-associated proteins in HepG2 and L02 cells were detected by western blotting. The results showed that higher concentrations of SWNHs significantly increased SIRT3 expression in HepG2 cells, especially in SW40 and SW50. SIRT3 works as a tumor suppressor that is localized in the mitochondria, which is able to maintain the integrity of mitochondria, metabolism during stress (8) and help to depress mitochondrial membrane permeability. Numerous studies reported that the upregulation of SIRT3 might be able to maintain the integrity of the mitochondrial membrane and have a protective role in normal cells $(6,7,9)$. However, the upregulation of SIRT3 has different effects on tumor cells. A number of studies have indicated that SIRT3 was able to inhibit the growth and proliferation of HepG 2 cells as well as inducing apoptosis, which may be associated with the upregulation of MnSOD and p53. Furthermore, Bax and Fas were upregulated by MnSOD (36,37). Meanwhile, our results showed that SWNHs are also able to facilitate the expression of VDAC1 and CYT-C in HepG2 cells. VDAC1 is a key component of the MPTP, which is able to signal CYT-C to initiate the mitochondrial-mediated cell death cascade (38). The accumulation of SWNHs in cells are able to accelerate the expression of VDAC1, which increases mitochondrial permeability, upsets the voltage balance and causes MMP depolarization (39). Studies have revealed that the activity of AceCS2 contributes to the growth of cancer cells under conditions of hypoxia and lipid depletion (40). Interestingly, SWNHs therapy was able to attenuate AceCS2 expression via SIRT3 outbreak bursts $(27,28)$, which meant SWNHs are able to interfere with cellular tricarboxylic acid cycle and affect cellular energy metabolism.

These findings suggested that there are three ways of how SWNH treatment may result in HepG2 mitochondrial dysfunction-induced apoptosis (Fig. 6), where SIRT3 was a midpoint (34). Firstly, SWNH treatment inhibited the activity of AceCS2 via SIRT3, then blocked fatty acid synthesis and the tricarboxylic acid cycle. Secondly, the outbreak of SIRT3 induced a large production of pro-apoptotic factors, Bax, Fax and CYT-C, and an accumulation of these factors in the mitochondria. These pro-apoptotic factors are able to pass through the mitochondrial membrane into the cytoplasm via the VDAC1 channel and then induce apoptosis. Thirdly, it had been demonstrated that SIRT3 was able to activate p53, which has a pivotal role in tumor suppression (18). As for L02 cells, the current therapeutic doses of SWNHs did not result in significant changes in the levels of mitochondrial apoptotic proteins, therefore this treatment regimen may be safe to normal cells within a certain range.

An in vivo experiment was performed to further confirm the molecular mechanism of how SWNHs promote the apoptosis of HepG2 cells. Although the effect of SWNH treatment on HepG2 tumor xenografts remain at an early stage based on the cell morphology and the expression level of CYT-C, 
the current dose of SWNHs was sufficient to increase the expression of SIRT3, Bax and VDAC1. There are several reasons that may account for the differences observed between immunohistochemical staining and western blotting. Firstly, immunohistochemical staining and western blotting are semiquantitative methods for proteins, and they do not fully reflect the actual protein expression. Secondly, there might be an unequal distribution of SWNHs in tumor xenografts. Thirdly, the number of experimental animals used is small, and there might be differences between the animals. In addition, Bax, CYT-C, SCNN1A and VDAC1 were mainly located in the cytoplasm and cell membranes, while AceCS2 appeared in the cytoplasm and nucleus in all xenografts. As for SIRT3, it was mainly expressed in the cytoplasm in the control group. However, following treatment with SWNHs, SIRT3 expression was observed in the nuclei of tumor cells. In addition, staining with hematoxylin and eosin and Sirius red was performed. There was no significant difference in the morphology of the cells or nuclei and the distribution of collagen fibers between the two groups (Fig. 5C). From these findings, the mechanism of how SWNHs promote mitochondrial dysfunction-induced apoptosis was further confirmed. This therapeutic effect was likely to be associated with the activation of SIRT3. SWNH treatment had a stable effect on SIRT3 based on the immunohistochemical staining and western blotting results. SWNHs were able to intrinsically induce mitochondrial dysfunction in HepG2 cells and apoptosis.

Taken together, the present study demonstrated that SWNHs, as a potential antineoplastic agent, are able to enter hepatoblastoma cells and accumulate in the mitochondria, triggering the release of SIRT3 from mitochondria to cytoplasm, which interferes with mitochondrial function and initiates mitochondrial dysfunction-induced apoptosis.

\section{Acknowledgements}

Not applicable.

\section{Funding}

The present study was supported by grants from the Natural Science Foundation of China (grant no. 81641110), the Guangdong Province Natural Science Foundation (grant nos. 2015A030313725 and 2017A030313510) and the Introduction of Talent Fund of Guangdong Second Provincial General Hospital (grant no. YY2016-006).

\section{Availability of data and materials}

The analyzed data sets generated during the study are available from the corresponding author on reasonable request.

\section{Authors' contributions}

$\mathrm{BL}$ and $\mathrm{XC}$ conducted the cell and animal experiments and collected the data and wrote the manuscript. WY, JH, KH and $\mathrm{ZX}$ analyzed and interpreted the data from the experimental results. GX and JZ were involved in the conception and design of the study, and revised the manuscript. All authors have read and approved the final version of the manuscript.

\section{Ethics approval and consent to participate}

The experiments on the mice were performed according to the standards that were supported by the Animal Protection Committee of Southern Medical University. The ethics approval was obtained from Animal Ethical and Welfare Committee of South Medical University (reference no. IACUC-2017-0120).

\section{Patient consent for publication}

Not applicable.

\section{Competing interests}

The authors declare that they have no competing interests.

\section{References}

1. Bosch FX, Ribes J, Díaz M and Cléries R: Primary liver cancer: Worldwide incidence and trends. Gastroenterology 127 (Suppl 1): S5-S16, 2004.

2. Ross JA and Gurney JG: Hepatoblastoma incidence in the United States from 1973 to 1992. Med Pediatr Oncol 30: 141-142, 1998.

3. Fuchs J, Rydzynski J, Von Schweinitz D, Bode U, Hecker H, Weinel P, Bürger D, Harms D, Erttmann R, Oldhafer K, et al; Study Committee of the Cooperative Pediatric Liver Tumor Study Hb 94 for the German Society for Pediatric Oncology and Hematology: Pretreatment prognostic factors and treatment results in children with hepatoblastoma: A report from the German Cooperative Pediatric Liver Tumor Study HB 94. Cancer 95: 172-182, 2002.

4. Suita S, Tajiri T, Takamatsu H, Mizote H, Nagasaki A, Inomata $Y$, Hara T, Okamura J, Miyazaki S, Kawakami K, et al: Improved survival outcome for hepatoblastoma based on an optimal chemotherapeutic regimen - a report from the study group for pediatric solid malignant tumors in the Kyushu area. J Pediatr Surg 39: 195-198, 2004.

5. Reynolds M: Conversion of unresectable to resectable hepatoblastoma and long-term follow-up study. World J Surg 19: 814-816, 1995.

6. Claycombe KJ, Roemmich JN, Johnson L, Vomhof-DeKrey EE and Johnson WT: Skeletal muscle Sirt3 expression and mitochondrial respiration are regulated by a prenatal low-protein diet. J Nutr Biochem 26: 184-189, 2015.

7. Jiang DQ, Wang Y, Li MX, Ma YJ and Wang Y: SIRT3 in neural stem cells attenuates microglia activation-induced oxidative stress injury through mitochondrial pathway. Front Cell Neurosci 11: 7, 2017.

8. Kim HS, Patel K, Muldoon-Jacobs K, Bisht KS, Aykin-Burns N, Pennington JD, van der Meer R, Nguyen P, Savage J, Owens KM, et al: SIRT3 is a mitochondria-localized tumor suppressor required for maintenance of mitochondrial integrity and metabolism during stress. Cancer Cell 17: 41-52, 2010.

9. Sundaresan NR, Samant SA, Pillai VB, Rajamohan SB and Gupta MP: SIRT3 is a stress-responsive deacetylase in cardiomyocytes that protects cells from stress-mediated cell death by deacetylation of Ku70. Mol Cell Biol 28: 6384-6401, 2008.

10. Qiao A, Wang K, Yuan Y, Guan Y, Ren X, Li L, Chen X, Li F, Chen AF and Zhou J: Sirt3-mediated mitophagy protects tumor cells against apoptosis under hypoxia. Oncotarget 7: 43390-43400, 2016.

11. Qiu X, Brown K, Hirschey MD, Verdin E and Chen D: Calorie restriction reduces oxidative stress by SIRT3-mediated SOD2 activation. Cell Metab 12: 662-667, 2010.

12. Haigis MC, Deng CX, Finley LWS, Kim HS and Gius D: SIRT3 is a mitochondrial tumor suppressor: A scientific tale that connects aberrant cellular ROS, the Warburg effect, and carcinogenesis. Cancer Res 72: 2468-2472, 2012.

13. Iijima S, Yudasaka M, Yamada R, Bandow S, Suenaga K, Kokai F and Takahashi K: Nano-aggregates of single-walled graphitic carbon nano-horns. Chem Phys Lett 309: 165-170, 1999.

14. Murakami T and Tsuchida K: Recent advances in inorganic nanoparticle-based drug delivery systems. Mini Rev Med Chem 8: 175-183, 2008. 
15. Chakrabarti M, Kiseleva R, Vertegel A and Ray SK: Carbon nanomaterials for drug delivery and cancer therapy. J Nanosci Nanotechnol 15: 5501-5511, 2015.

16. Cao Y, Zhang Y and Zhao M: Single-walled carbon nanohorns inhibit proliferation of conjunctival melanoma cell lines CRMM-1 and involved in energy metabolism. J Nanosci Nanotechnol 15: 1821-1830, 2015.

17. Li L, Zhang J, Yang Y, Wang Q, Gao L, Yang Y, Chang T, Zhang X, Xiang G, Cao Y, et al: Single-wall carbon nanohorns inhibited activation of microglia induced by lipopolysaccharide through blocking of Sirt3. Nanoscale Res Lett 8: 100, 2013.

18. Zhang J, Sun Q, Bo J,Huang R, Zhang M, Xia Z, Ju L and Xiang G: Single-walled carbon nanohorn (SWNH) aggregates inhibited proliferation of human liver cell lines and promoted apoptosis, especially for hepatoma cell lines. Int J Nanomedicine 9: 759-773, 2014

19. Datler C, Pazarentzos E, Mahul-Mellier AL, Chaisaklert W, Hwang MS, Osborne F and Grimm S: CKMT1 regulates the mitochondrial permeability transition pore in a process that provides evidence for alternative forms of the complex. J Cell Sci 127: 1816-1828, 2014.

20. Zamzami $\mathrm{N}$ and Kroemer G: The mitochondrion in apoptosis: How Pandora's box opens. Nat Rev Mol Cell Biol 2: 67-71, 2001.

21. Susin SA, Zamzami N and Kroemer G: Mitochondria as regulators of apoptosis: Doubt no more. Biochim Biophys Acta 1366 $151-165,1998$

22. Cotter TG, Lennon SV, Glynn JG and Martin SJ: Cell death via apoptosis and its relationship to growth, development and differentiation of both tumour and normal cells. Anticancer Res 10: 1153-1159, 1990

23. Wong RS: Apoptosis in cancer: from pathogenesis to treatment. J Exp Clin Cancer Res 30: 87, 2011.

24. Ventura-ClapierR,Moulin M,Piquereau J,Lemaire C,Mericskay M, Veksler V and Garnier A: Mitochondria: A central target for sex differences in pathologies. Clin Sci (Lond) 131: 803-822, 2017.

25. Burke PJ: Mitochondria, bioenergetics and apoptosis in cancer. Trends Cancer 3: 857-870, 2017.

26. Zsíros J, Maibach R, Shafford E, Brugieres L, Brock P, Czauderna P, Roebuck D, Childs M,Zimmermann A, Laithier V, et al: Successful treatment of childhood high-risk hepatoblastoma with doseintensive multiagent chemotherapy and surgery: Final results of the SIOPEL-3HR study. J Clin Oncol 28: 2584-2590, 2010.

27. Pritchard J, Brown J, Shafford E, Perilongo G, Brock P, Dicks-Mireaux C, Keeling J, Phillips A, Vos A and Plaschkes J: Cisplatin, doxorubicin, and delayed surgery for childhood hepatoblastoma: A successful approach - results of the first prospective study of the International Society of Pediatric Oncology. J Clin Oncol 18: 3819-3828, 2000.

28. Perilongo G, Shafford E and Plaschkes J; Liver Tumour Study Group of the International Society of Paediatric Oncology: SIOPEL trials using preoperative chemotherapy in hepatoblastoma. Lancet Oncol 1: 94-100, 2000.
29. von Schweinitz D: Hepatoblastoma: Recent developments in research and treatment. Semin Pediatr Surg 21: 21-30, 2012.

30. Liu ZB, Zhou B, Wang HY, Zhang HL, Liu LX, Zhu DW and Leng XG: Effect of functionalized multi-walled carbon nanotubes on L02 cells. Zhongguo Yi Xue Ke Xue Yuan Xue Bao 32: 449-455, 2010 (In Chinese).

31. Matsuda S, Matsui S, Shimizu Y and Matsuda T: Genotoxicity of colloidal fullerene $\mathrm{C}_{60}$. Environ Sci Technol 45: 4133-4138, 2011

32. Zhou F, Xing D, Wu B, Wu S, Ou Z and Chen WR: New insights of transmembranal mechanism and subcellular localization of noncovalently modified single-walled carbon nanotubes. Nano Lett 10: 1677-1681, 2010.

33. Ajima K, Murakami T, Mizoguchi Y, Tsuchida K, Ichihashi T, Iijima $S$ and Yudasaka M: Enhancement of in vivo anticancer effects of cisplatin by incorporation inside single-wall carbon nanohorns. ACS Nano 2: 2057-2064, 2008.

34. Zhang JY, Deng YN, Zhang M, Su H and Qu QM: SIRT3 acts as a neuroprotective agent in rotenone-induced Parkinson cell model. Neurochem Res 41: 1761-1773, 2016.

35. Iijima T: Mitochondrial membrane potential and ischemic neuronal death. Neurosci Res 55: 234-243, 2006.

36. Liu Y, Liu YL, Cheng W, Yin XM and Jiang B: The expression of SIRT3 in primary hepatocellular carcinoma and the mechanism of its tumor suppressing effects. Eur Rev Med Pharmacol Sci 21: 978-998, 2017

37. Tao R, Vassilopoulos A, Parisiadou L, Yan Y and Gius D: Regulation of MnSOD enzymatic activity by Sirt3 connects the mitochondrial acetylome signaling networks to aging and carcinogenesis. Antioxid Redox Signal 20: 1646-1654, 2014.

38. Barbieri E, Battistelli M, Casadei L, Vallorani L, Piccoli G, Guescini M, Gioacchini AM, Polidori E, Zeppa S, Ceccaroli P, et al: Morphofunctional and biochemical approaches for studying mitochondrial changes during myoblasts differentiation. J Aging Res 2011: 845379, 2011

39. Maldonado EN and Lemasters JJ: Warburg revisited: Regulation of mitochondrial metabolism by voltage-dependent anion channels in cancer cells. J Pharmacol Exp Ther 342: 637-641, 2012.

40. Schug ZT, Peck B, Jones DT, Zhang Q, Grosskurth S, Alam IS Goodwin LM, Smethurst E, Mason S, Blyth K, et al: Acetyl-CoA synthetase 2 promotes acetate utilization and maintains cancer cell growth under metabolic stress. Cancer Cell 27: 57-71, 2015.

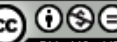

This work is licensed under a Creative Commons Attribution-NonCommercial-NoDerivatives 4.0 International (CC BY-NC-ND 4.0) License. 